\title{
A COMBINATORIAL THEOREM FOR STOCHASTIC PROCESSES
}

\section{BY LAJOS TAKÁCS ${ }^{1}$}

Communicated by J. L. Doob, January 21, 1965

Let $\{\chi(u), 0 \leqq u \leqq t\}$ be a stochastic process where $t$ is a finite positive number. We associate a stochastic process $\left\{\chi^{*}(u), 0 \leqq u<\infty\right\}$ with $\{\chi(u), 0 \leqq u \leqq t\}$ as follows: $\chi^{*}(u)=\chi(u)$ for $0 \leqq u \leqq t$ and $\chi^{*}(t+u)=\chi^{*}(t)+\chi^{*}(u)$ for $u>0$. If the finite dimensional distributions of $\left\{\chi^{*}(v+u)-\chi^{*}(v), 0 \leqq u \leqq t\right\}$ are independent of $v$ for $v \geqq 0$, then the process $\{\chi(u), 0 \leqq u \leqq t\}$ is said to have cyclically interchangeable increments. In particular, if $\{\chi(u), 0 \leqq u \leqq t\}$ has stationary, independent increments, and $P\{\chi(0)=0\}=1$, then it belongs to this class.

Theorem. If $\{\chi(u), 0 \leqq u \leqq t\}$ is a separable stochastic process with cyclically interchangeable increments and if almost all sample functions are nondecreasing step functions which vanish at $u=0$, then

$$
P\{\chi(u) \leqq u \text { for } 0 \leqq u \leqq t \mid \chi(t)\}
$$

$$
=\left\{\begin{array}{cl}
\left(1-\frac{\chi(t)}{t}\right) & \text { if } 0 \leqq \chi(t) \leqq t \\
0 & \text { otherwise, }
\end{array}\right.
$$

with probability 1.

Proof. Let $\chi^{*}(u), 0 \leqq u<\infty$, be a nondecreasing step function (nonrandom) for which $\chi^{*}(0)=0$ and $\chi^{*}(t+u)=\chi^{*}(t)+\chi^{*}(u)$ if $u>0$ where $t$ is a fixed positive number. For $u \geqq 0$ define

$$
\xi(u)= \begin{cases}1 & \text { if } \chi^{*}(v)-\chi^{*}(u) \leqq v-u \text { for } v \geqq u, \\ 0 & \text { otherwise. }\end{cases}
$$

Obviously $\xi(u+t)=\xi(u)$ for all $u \geqq 0$. Now we shall prove that

$$
\int_{0}^{t} \xi(u) d u=\left\{\begin{array}{cl}
t-\chi^{*}(t) & \text { if } 0 \leqq \chi^{*}(t) \leqq t \\
0 & \text { otherwise. }
\end{array}\right.
$$

The case $\chi^{*}(t) \geqq t$ is obvious. Thus we suppose that $0 \leqq \chi^{*}(t)<t$. Define

1 This research was sponsored by the Office of Naval Research under Contract Number Nonr-266(59). 


$$
\alpha(c)=\sup \left\{u: u-\chi^{*}(u)<c \text { and } 0 \leqq u<\infty\right\}
$$

for $c>0$. The function $\alpha(c)$ is increasing. $\alpha(c)$ increases either linearly with slope 1 or by jumps. Evidently $\alpha(c-0)=\alpha(c)$ and $\alpha\left(c+t-\chi^{*}(t)\right)$ $=\alpha(c)+t$ for $c>0$. If $u=\alpha(c)$, then $\xi(u)=1$. Since $\alpha(c)-\chi^{*}(\alpha(c))=c$ and $u-\chi^{*}(u) \geqq c$ for $u \geqq \alpha(c)$, it follows that $\chi^{*}(u)-\chi^{*}(\alpha(c)) \leqq u-\alpha(c)$ for $u \geqq \alpha(c)$, that is, $\xi(\alpha(c))=1$. If $\alpha(c)<u<\alpha(c+0)$, then evidently $\xi(u)=0$. Accordingly, in the interval $\left[\alpha(c), \alpha\left(c+t-\chi^{*}(t)\right)\right]$ of length $t, \xi(u)=1$ on the set $\left\{u: u=\alpha(z)\right.$ for $\left.c \leqq z \leqq c+t-\chi^{*}(t)\right\}$ of measure $t-\chi^{*}(t)$ and $\xi(u)=0$ elsewhere. (If $\chi^{*}(u)$ is defined as continuous on the right, and $z$ is a discontinuity point of $\alpha(z)$, then also $\xi(\alpha(z+0))$ $=1$. However, since the discontinuity points of $\alpha(z)$ form a set of measure 0 , this does not make any difference.) Since $\xi(u)$ is periodic with period $t$, (3) follows.

Now, if we suppose that $\left\{\chi^{*}(u), 0 \leqq u<\infty\right\}$ is the stochastic process associated with the process $\{\chi(u), 0 \leqq u \leqq t\}$ under consideration and if $\xi(u)$ is defined by (2), then $\xi(u)$ is a random variable which has the same distribution for all $u \geqq 0$. Thus

$$
\begin{aligned}
P\{\chi(u) & \leqq u \text { for } 0 \leqq u \leqq t \mid \chi(t)\} \\
& =E\{\xi(0) \mid \chi(t)\}=\frac{1}{t} \int_{0}^{t} E\{\xi(u) \mid \chi(t)\} d u \\
& =E\left\{\frac{1}{t} \int_{0}^{t} \xi(u) d u \mid \chi(t)\right\}=\left\{\begin{array}{cc}
\left(1-\frac{\chi(t)}{t}\right) & \text { if } 0 \leqq \chi(t) \leqq t, \\
0 & \text { otherwise, }
\end{array}\right.
\end{aligned}
$$

with probability 1 . The last equality follows from identity (3) which now holds for almost all sample functions. This completes the proof of the theorem.

COLUMBIA UNIVERSITY 